\title{
Laser physics: Quantum controversy in action
}

\author{
W. E. Lamb \\ Optical Sciences Center, University of Arizona, Tucson, Arizona, 85721 \\ W. P. Schleich \\ Abteilung für Quantenphysik, Albert-Einstein Allee 11, Universität Ulm, \\ D-89069 Ulm, Germany \\ M. O. Scully \\ Department of Physics, Texas A\&M University, College Station, Texas 77843 \\ and Max-Planck-Institut für Quantenoptik, Hans-Kopfermann Straße 1, \\ D-85748 Garching, Germany

\section{H. Townes} \\ Department of Physics, University of California at Berkeley, Berkeley, California 94720
}

We summarize the history and discuss quantum subtleties of maser/laser physics from early days until the present. [S0034-6861(99)03302-4]

\section{CONTENTS}

I. Introduction

II. Maser Prehistory

A. The search for the perfect oscillator

B. "Negative absorption"

C. Stimulated emission: Einstein and Dirac versus Maxwell

III. The Maser-How It Came To Be

A. From water vapor and radar to microwave spectroscopy

B. The idea on the park bench

C. The birth of the maser

D. Why not earlier?

IV. On the Road Towards the Laser
A. Early maser research
B. Why lasers will not work
C. Lamb dip

V. Quantum Effects in Masers and Lasers
A. Linewidth
B. Squeezing and the correlated spontaneous emission laser
C. Photon statistics
D. One-atom masers/lasers and the mazer
E. The laser phase transition analogy

VI. Exotic Lasers and Outlook
A. The free-electron laser
B. Lasers without inversion
C. The atom laser

Acknowledgments

References

\section{INTRODUCTION}

Laser physics is a subject whose roots go back to the very source of quantum thought, namely, blackbody radiation and the Planck distribution. But the road to the first maser device showing radiation amplification by stimulated emission was rocky indeed. Likewise, the struggle to produce the first laser was hampered by much false "wisdom." Furthermore, the understanding of the laser, its extraordinary radiation, and its limits of operation is a great example of how our understanding of quantum physics has developed through and is illuminated by vigorous argument and debate, which extends even unto today.

For example, the very nature of our understanding of light (photon-wave) is a matter of much discussion and misunderstanding. We shall endeavor to present maser/ laser physics from the prospective of quantum controversy; looking at the pros and cons of various issues, false starts and key clues. This, after all, is the way physics develops, moving forward in the real-life give-andtake of debate, and often, intense controversy.

In the first section we briefly review the premaser days and highlight some stepping stones towards the maser and laser. In particular, we focus on the basic underpinning of laser physics: stimulated emission. The next two sections present the development of maser/laser science and the semiclassical theory of its operation. However, the many subtle features of the laser come to light only within the quantum theory of the laser. This is the topic of the next section. We conclude by presenting a few exotic laser concepts.

We emphasize that there are many more exciting developments in laser physics than we can cover in this review. For example, space does not allow us to discuss in detail the field of semiconductor lasers and its theory. Nor can we but mention the fields of excimer or x-ray lasers. Femto-second pulses have opened the new field of wave-packet dynamics and femto-chemistry, which represents another frontier of laser science that we can only allude to in this article. Nevertheless, these examples illustrate in a vivid way that laser physics is alive and well, both as a scientific and engineering discipline as we enter the 21 st century.

\section{MASER PREHISTORY}

Conventional wisdom associates the beginning of the maser/laser with Einstein's discovery of the phenomenon of stimulated emission; even the acronym of 
microwave/light amplification by stimulated emission radiation suggests this. However, in the spirit of the title of this paper, Quantum Controversy, we argue that the path leading to these devices started even earlier.

\section{A. The search for the perfect oscillator}

When God said "let there be light" he surely must have meant perfectly coherent light, that is, a perfect oscillator. But how to create such a perfect oscillator? Start from a source of dc energy for an oscillator; then by some trick, change the dc into ac: the result is a selfsustained oscillator.

The most elementary example is a grandfather clock. Here, we turn a knob, and raise a weight. Its potential energy is converted into periodic swinging of the pendulum bob. Moreover, a ratchet serves as a control mechanism between the super-hot reservoir-that is, the weight in the gravitational field - and the bob system.

Another example, from the realm of acoustical oscillators, is the aolean harp known since the time of King David. In a paper reprinted in his collected works, Lord Rayleigh reports about an experiment in which he has a steady stream of wind blowing across the strings of a harp, creating sound. This is similar to the "Galloping Gertie," which is the oscillating Tacoma Narrows Bridge that in the 1940s was swinging for days and finally collapsed. These are three examples in which a steady source of energy creates a self-sustained oscillator.

Why not mention in the domain of electronics the triode vacuum-tube radio-frequency oscillator developed by L. De Forest in 1912? This was, in fact, the first electrical oscillator made by man. In 1921 E.V. Appleton and B. Van der Pol gave a classical theory of this device. It introduced for the first time the concept of "negative resistance." We can think of the negative resistance as a source of dc energy that can drive oscillations. For example, two-level atoms with a population inversion are a source of energy that can drive "laser" oscillations.

Later, in his book Principles of Electricity and Magnetism, Gaylord P. Harnwell (1938) gave a simple model for a de Forrest triode oscillator. He showed that a nonlinear negative resistance in an RLC circuit can generate self-sustained oscillations. If the applied voltage is zero, and the circuit has a little noise, the oscillations build up from noise to a steady state. Here, the effective resistance becomes zero. For large currents, the effective resistance becomes positive instead of negative. This leads to saturation. This is very similar to the laser.

In the late 1930s, two brothers, Russell and Sigurd Varian $^{1}$, invented the klystron oscillator at Stanford.

\footnotetext{
${ }^{1}$ Russell Varian was a physicist and considered a genius. However, he did not hold a Ph.D. although he was awarded honorary degrees later on. His brother Sigurd Varian was a professional airplane pilot and was therefore interested in short-wave radio communications. Eventually, the brothers founded a company, Varian Associates, that produced klystron devices and many instruments for scientific and commercial purposes.
}

Here, a dc electron beam passes through two cavities. The first cavity is called buncher, and the second cavity is called catcher, which is where the electromagnetic oscillations build up. There is feedback from the catcher to the buncher.

Another electromagnetic oscillator is the magnetron, developed in 1920 by Albert W. Hull. It is like a triode oscillator or a klystron. It is fully classical in operation, except it is also made of electrons and atoms, which need quantum mechanics. Magnetrons and klystrons have played a central role in war research related to radar. It was this radar work that laid the path for the maser as we discuss in the next section.

\section{B. "Negative absorption"}

Intimately related to the concept of "negative resistance," discussed in the preceding section, are the ideas of "negative absorption" and "population inversion." They occur already in various early publications on atomic spectroscopy and constitute important stepping stones on the path to the maser and laser. Since space does not allow us to discuss all these papers in detail we shall highlight only a few.

In $1924 \mathrm{R}$. Tolman wrote in a long article on an excited gaseous medium:

"The possibility arises, however, that molecules in the upper quantum state may return to the lower quantum state in such a way as to reinforce the primary beam by 'negative absorption'..."

However, he concluded that

“... for absorption experiments as usually performed, the amount of 'negative absorption' can be neglected."

Two years later an interesting episode took place. The chemist G.N. Lewis in Berkeley proposed in a paper entitled "The conservation of photons" a mechanism for chemical bonding. The particle that achieves this he called photon. He certainly meant something completely different from Einstein's light quantum. The word "photon" caught on but not his meaning (Lamb, 1995).

Rudolf Walther Ladenburg played an important role in the history of the maser and laser. He came very close to discovering amplification by stimulated emission. Indeed, in the 1920s, while he was still in Germany, Ladenburg performed experiments with his co-workers (most prominently Hans Kopfermann), on the dispersion of gaseous neon near the red emission lines. Neon was excited in a tube by means of an electric discharge and the dispersion was studied as a function of the discharge current density. As Ladenburg summarized his experiments in 1933:

"...the experiments prove the influence of the negative term in the dispersion formula. This "negative dispersion" corresponds to the negative absorption of the theory of radiation..." 
The theme of negative absorption reoccurs in the context of the fine structure of the hydrogen atom (Lamb and Retherford, 1950). The authors show that if the state

“... $2^{2} P_{3 / 2}$ is more highly populated, there will be a net induced emission (negative absorption!)."

We conclude this discussion of the prehistory of the maser by briefly mentioning the work of V. A. Fabrikant in the former Soviet Union. In his thesis in 1940 he also discussed the consequences of population inversion.

\section{Stimulated emission: Einstein and Dirac versus Maxwell}

In his derivation of the Planck radiation formula in 1917 Albert Einstein introduced the $A$ coefficient for the rate of spontaneous emission by atoms and the $B$ coefficient for their absorption of radiation. He also introduced the new process of stimulated emission of radiation and found that the $B$ coefficient determined its rate. Ten years later the quantum electrodynamics (QED) of P. A. M. Dirac provided the deeper foundation.

However, Einstein's result is perfectly natural when we disregard, for a moment, Maxwell's electromagnetic theory and, instead, believe in the 1905 concept of photons and in the Bohr orbits. Then it is natural to have spontaneous emission and absorption of the light particles, and the new feature is, indeed, stimulated emission. However, we emphasize that Maxwell's theory also predicts these phenomena.

To bring this out most clearly, we consider a charged particle oscillating back and forth in an electromagnetic wave. We recall that a particle of charge $q$ moving with velocity $v$ in an electric field $E$, gains or loses energy depending on the algebraic sign of the product $q E v$. An increase of the energy of the charge implies a loss of energy in the field. This is equivalent to the process of absorption of radiation. Likewise, if the charge is losing energy, the electromagnetic field must be gaining energy. This is equivalent to stimulated emission of radiation. The relative direction of the velocity and the electric-field vectors determines the direction of the energy flow between field and matter (Lamb, 1960). Moreover, the fact that an accelerated charge radiates corresponds to the process of spontaneous emission.

How does this translate into the language of QED? To answer this question, we consider the change of the electromagnetic field due to the transition of an excited atom into its ground state. We assume that initially only one mode is occupied by $n$-quanta and all the other modes are empty. The atomic transition creates one quantum of field excitation in any field mode. However, due to the property

$$
\hat{a}^{\dagger}|n\rangle=\sqrt{n+1}|n+1\rangle
$$

of the creation operator, the mode with $n$-quanta already present has a higher probability compared to the vacuum modes where $n=0$. Hence the amplification, which is stimulated emission, is preferentially in the mode of the incident radiation.

But how can we use Maxwell's theory to explain the directionality of the emitted radiation, which is so obvious in the QED formulation? On first sight this seems to be impossible: A dipole does not radiate in the direction in which it is driven. However, when we calculate the energy flow, that is, the Poynting vector of the total field consisting of the incident and the radiated electromagnetic field, the interference term between the two provides the directionality. Indeed, this term is rapidly oscillating in space except along a narrow cone along the axis of propagation of the incident radiation (Sargent et al., 1974).

We conclude this section by briefly alluding to one more feature of stimulated emission. Stimulated emission is said to be in phase with the incident radiation. We can understand this feature when we recall that the induced dipole is a driven oscillator. Therefore it is in phase and has the same frequency as the incident lightthere is no way to see this easily from QED!

\section{THE MASER-HOW IT CAME TO BE}

In the present section we briefly follow the path from the early work on microwave absorption in water vapor to the conception and realization of the ammonia maser. We also speculate why the maser was not discovered earlier.

\section{A. From water vapor and radar to microwave spectroscopy}

The absorption of microwave radiation in water vapor was an important question during W.W. II. Indeed, it was recognized by J. H. Van Vleck and V. F. Weisskopf (1945) that the shortest waves (K band) might be absorbed in water vapor. If correct, this would have drastically reduced the use of radar in the South Pacific, an area of high rainfall. Therefore Isidor I. Rabi got authorization to study this question at the Columbia Radiation Laboratory at Columbia University.

Willis E. Lamb was involved in this research directed by J. M. Kellogg. Water vapor was inside a $8 \mathrm{ft} . \times 8 \mathrm{ft} . \times 8$ ft. resonator made of copper sheet. Large rotating copper fans "mixed up" the mode structure of the $1 \mathrm{~cm}$ microwaves in this resonator. The room was heated with steam radiators, to simulate tropical conditions. The experiments (Becker and Autler, 1946) indicated that there was some microwave absorption, but not bad enough to give up on the K-band completely (Lamb, 1946). As Lamb recalls those years in the labs:

"I learned something from this work, although not enough to invent microwave ovens. But I might have been the first to warm up cold hamburgers or coffee using centimeter microwave radiation. That came in handy for lunch." 
During the war Charles H. Townes worked at Bell labs on radar bombing and navigation systems. He also studied Van Vleck's work, and argued strongly that water vapor would have a disastrous effect on K-band radar in the Pacific arena. But after contacting several people about it, high-level officials simply told him the decision had been made to proceed and could not be changed.

In the process of examination and argument, Townes recognized that microwave absorption by such molecules at low pressures could provide a new kind of high-resolution spectroscopy (Townes, 1946). The new field largely used components of the K-band radar, which were in surplus because that wavelength was indeed relatively useless for radar. About ten years earlier, Claude E. Cleeton and Neil H. Williams (1934) at the University of Michigan had studied absorption of microwaves by the inversion of ammonia molecules, an absorption which had been predicted by David Dennison (1932) and colleagues at their university. This was a striking demonstration of the inversion of ammonia, a resonance which was later to be the basis for the first masers. However, they used ammonia at atmospheric pressure which gave absorption over a range almost as large as the frequency itself, not the high-resolution-atlow-pressure characteristic of postwar microwave spectroscopy.

\section{B. The idea on the park bench}

As the field of microwave spectroscopy progressed, Townes grew increasingly eager to extend it into the millimeter and submillimeter wavelengths, where absorption would be still stronger. He worked on several possible methods to achieve this, including Cerenkov radiation, magnetron harmonics, and electron-spin resonances. Knowing of his interest, the Office of Naval Research asked him to head a national committee to explore how shorter-wavelength oscillators might be achieved.

After about 18 months of committee discussions and visits to laboratories in the field, a final meeting was held in Washington in the spring of 1951 . Townes was frustrated that no great ideas had turned up and, waking up early before the committee meeting, left his hotel and sat down on a park bench. In musing over the problem and his frustrations with it, he suddenly recognized that molecules could produce much more than thermal radiation intensities if they were not thermally distributed but had more molecules or atoms in an upper than in a lower state. Within about ten minutes he had invented such a system using a beam of ammonia and a cavity, and calculated that it seemed practical to get enough molecules to cross the threshold of oscillation. This meant that molecular-stimulated emission at a given radiation intensity would be greater than energy loss in the walls of the cavity.

He did not know that an eventual outcome would be fantastic communication by optical fibers, nor did he know at the time that he was sitting next to the building where Alexander Graham Bell had worked for a long time trying to successfully communicate with light waves. However, the fact that the new idea occurred right next to Bell's old laboratory may now seem a bit mystical.

\section{The birth of the maser}

Townes's initial plan was to use ammonia rotational levels and work in the far-infrared or submillimeter region. But the $\mathrm{K}$-band region, or centimeter wavelengths and the ammonia inversion spectrum, seemed an easier start. So, after a few months, and carefully checking the coherence of stimulated emission using his notes on the quantum theory of radiation from his student days, Townes got together with an excellent student and postdoc, James Gordon and Herbert Zeiger, and set to work on the new type of oscillator.

However, as Townes (1999) recalls, his maser team at the Columbia Radiation Lab did not get much encouragement.

"One day, after we had been at it for about two years, Rabi and Kusch, the former and current chairmen of the department, both of them Nobel Laureates for their work with atomic and molecular beams and with a lot of weight behind their opinions, came into my office and sat down. They were worried. Their research depended on support from the same source as did mine. "Look," they said, "you should stop the work you are doing. You're wasting money. Just stop!"

At this moment Townes was indeed thankful that he came to Columbia with tenure already.

One of the problems that almost prevented the birth of the maser was the worry about too much radiation leaking through the entrance and the exit holes in the cavity for the molecular beam. Various metal rings designed to keep the microwaves inside of the resonator were tried. No success! Only when Jim Gordon, skipping a seminar, opened the ends almost completely did the device work (Gordon et al., 1954, 1955). As Townes recalls:

"The first maser had been born. This was about three months after Poly Kusch had insisted it would not work. But when it worked, he was gracious about it, commenting that he should have realized I probably knew more about what I was doing than he did."

\section{Why not earlier?}

Probably the reason why quantum electronics, that is, masers and lasers, did not develop sooner is that some aspects of the maser are most easily envisioned from a classical point of view and stimulated by an interest in electronics, while others require an understanding of quantum mechanics. In the early days of the field, it was clear that electrical engineers intuitively understood 
some characteristics of the maser oscillator much better than many physicists, who usually thought in terms of photons. However, at that time few engineers understood stimulated emission.

That a background in microwave spectroscopy, with a combination of engineering and quantum mechanics, was important to putting together appropriate concepts of useful amplification by stimulated emission has empirical support. The general idea appeared at approximately the same time to three groups: Joseph Weber of the University of Maryland (1953), Nickolai Basov and Alexander Prokhorov of the Lebedev Institute (1955), and Charles Townes at Columbia (Gordon et al., 1954), all of whom were working on microwave spectroscopy.

\section{ON THE ROAD TOWARDS THE LASER}

In the present section we discuss the development of the laser. In particular, we review the basic obstacles lying in the path of this development and discuss their solutions.

\section{A. Early maser research}

Interest in masers grew rapidly, partly because of their value as frequency standards, and partly because as amplifiers, they could be one or two orders of magnitude more sensitive than other types available. The spectral width of maser oscillators in the presence of thermal noise was derived by Gordon et al. (1955). A more quantum-mechanical approach to maser oscillation was developed by Shimoda et al. (1956), which gave spectral widths when dominated by quantum emission. A quantum-mechanical theory of noise in maser (or laser) amplifiers was given by Shimoda et al. (1957). For amplification, electron-spin resonances in solids (Combrisson et al., 1956) and particularly 3-level systems (Bloembergen, 1956) were especially attractive since they could be fairly broad-band and tunable. Application of the uncertainty principle to complementary uncertainties in the phase of a wave and the number of quanta $\Delta n \Delta \theta$ $\geqslant 1 / 2$ was also soon discussed (Serber and Townes, 1960), along with the possibility of measuring the phase more accurately than the normal precision, which, with $\Delta n=\sqrt{\bar{n}}$, is $\Delta \theta \geqslant 1 / 2 \sqrt{\bar{n}}$.

\section{B. Why lasers will not work}

With a working maser in hand, thoughts naturally turned to the possibility of an optical maser. Townes recalls that many colleagues argued that the maser would not work at shorter wavelengths. An illustration of this skepticism is a 1958 report for the U.S. Air Force on technology which might be of importance in the following 25 years. Townes was a member of a committee to write the report in the summer of 1957 . He persuaded the group to mention not only the development of masers in the microwave region, but also the possibility of extending them to shorter wavelengths. The report was not issued in 1957, and a further study was made to com- plete it in the summer of 1958 by the same group except that Townes was absent. That was the same summer Schawlow and Townes (1958) finished and began to circulate their paper on "Optical Masers." But the committee, which had not seen the paper, did not accept Townes's previous recommendation: The committee decided to remove any mention of the possibility of extending masers to shorter wavelengths, and included only microwave masers as the technology of the next 25 years.

One of the many reasons for this early pessimism was that the rate of energy radiated spontaneously from a molecule increases as the fourth power of the frequency, assuming other characteristics of the molecule remain generally the same. Townes's original goal was to amplify at a wavelength of, say, one tenth of a millimeter instead of one centimeter. Hence to keep the electrons or molecules excited in a regime, this would require an increase in the pumping power by many orders of magnitude.

Another "problem" was that for gas molecules or atoms, Doppler effects increasingly broaden the emission spectrum as the frequency goes up. This leaves less amplification per molecule to drive any specific resonant frequency. For a description of this battle with the Doppler effect, see Lamb (1984).

Finally, there are no nice "cavities" for light. Indeed, the maser operating at wavelengths of the order of a centimeter can oscillate in a cavity whose length is equal to the wavelength. However, it is difficult to make similar cavities much smaller than a millimeter and virtually impossible to make them as small as an optical wavelength. A cavity related to the Fabry-Perot etalon or interferometer, whose length is many times the wavelength of resonance is the solution of the opticalresonator problem. This was pointed out by Schawlow and Townes (1958); a summarizing discussion is given by Siegman (1986).

Nothing stops "naysayers" like a working device. Theodore Maiman (1960) gave the first working laser to us-the pulsed ruby system. This was soon followed by other types of lasers, including those using gaseous discharges (Javan et al., 1961) and semiconductors (Basov et al., 1959 and Hall et al., 1962).

\section{Lamb dip}

Motivated by the experiments on the ammonia maser and building on his theoretical work on water-vapor absorption, Lamb, during the years 1954-1956, developed a theory of the maser (see Lamb, 1960). Later, he worked out a complete semiclassical theory of laser action (Lamb, 1964a, 1964b). It is based on a selfconsistent treatment of the polarization of the masing/ lasing medium as it drives the electric-field oscillator in the laser cavity. This semiclassical description of laser operation shown in Fig. 1 has been a touchstone of laser physics over the years.

We conclude by returning for a moment to the Doppler broadening problem. It turned out to be a blessing 


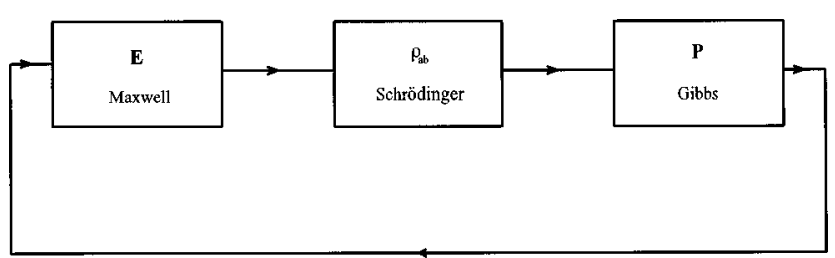

FIG. 1. Semiclassical theory of the laser as a self-consistent analysis. We start with a nonvanishing seed electric field $\mathbf{E}$ in the laser cavity. This field induces microscopic dipole moments in the active medium according to the laws of quantum mechanics. These moments represented by off-diagonal elements $\rho_{a b}$ of the density operator sum up statistically through a Gibbs ensemble to a macroscopic polarization $\mathbf{P}$. This generates via Maxwell's equations a new electric field $\mathbf{E}^{\prime}$. The condition of self-consistency requires that the seed field is equal to the generated field, that is, $\mathbf{E}=\mathbf{E}^{\prime}$.

in disguise. As shown by the semiclassical theory, there is a dip in the laser power as a function of cavity detuning. This so-called Lamb dip, shown in Fig. 2, was verified experimentally by McFarlane et al. (1963) and Szöke and Javan (1963) and has proven to be very useful in building ultrastable lasers: It provides a narrow resonance allowing to lock lasers to the center of the dip.

\section{QUANTUM EFFECTS IN MASERS AND LASERS}

Laser theory has come a long way from the early approaches using birth and death equations (Shimoda et al., 1957) via the semiclassical theory of the laser to the fully quantized version. The three approaches to-

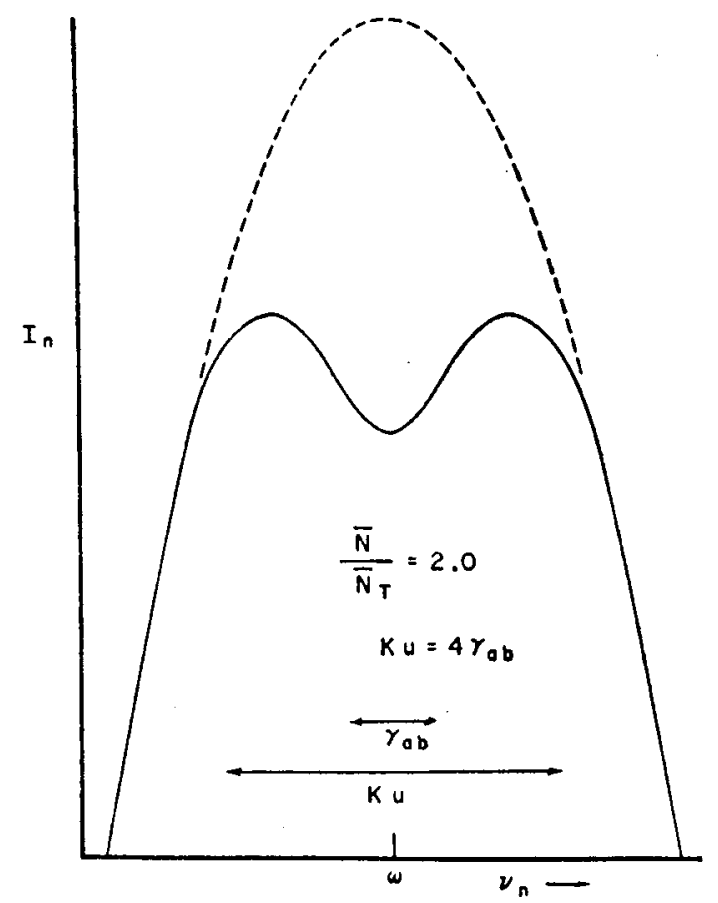

FIG. 2. Lamb dip. The laser intensity shows a characteristic dip as a function of cavity detuning. [From the original paper by Lamb (1964b)].
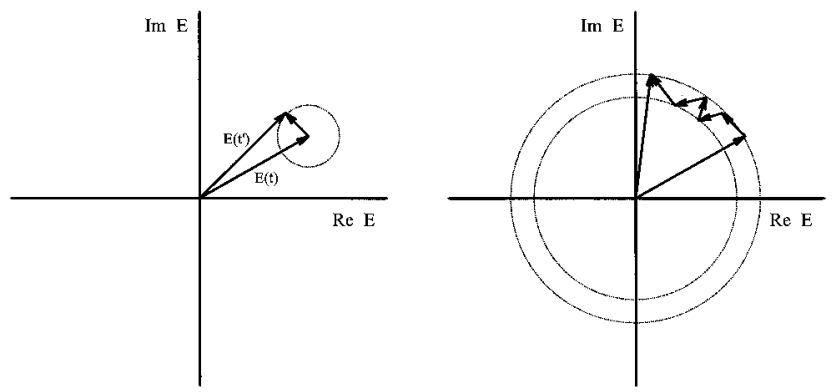

FIG. 3. Phase diffusion in a laser as a random walk in complex space. We represent the electric field of the laser by a vector in the complex plane. Its length is the amplitude of the field, whereas, its angle with the horizontal axis of the coordinate system is its phase. The spontaneous emission of an atom changes the electric field (left). The amplitude of the emitted field is small compared to the original field and its phase is completely uncorrelated. Due to many spontaneous emission events, the electric field undergoes Brownian motion in complex space (right). The saturation of the laser stabilizes the amplitude of the electric field and forces the phase to undergo diffusion in a band.

wards the quantum theory of the laser ${ }^{2}$ are the FokkerPlanck method (Haken and Risken), noise operators (Lax and Louisell), and density matrix techniques (Scully and Lamb). In the present section we now turn to the quantum aspects of laser light.

\section{A. Linewidth}

In order to study the laser linewidth (Schawlow and Townes, 1958) we represent the laser field by a vector in the complex plane as shown in Fig. 3. This vector describes the amplitude and the phase of the electromagnetic field. Strictly speaking, both are operators. However, in the present discussion it suffices to take them as fluctuating classical quantities. We emphasize that this replacement is not a trivial step and the question of the appropriate definition of a Hermitian phase operator is still a research topic (Schleich and Barnett, 1993).

Due to the spontaneous emission of the atom, the electric field experiences small changes and undergoes a random walk. Here, we assume that the small change takes place on a far shorter time scale than the overall evolution of the field. Moreover, we consider a situation in which the laser is operating sufficiently far above threshold so that the amplitude fluctuations can be ignored. The field $E(t)$ can then be written as

$$
E(t)=\sqrt{\bar{n}} e^{i \theta(t)} e^{-i \nu_{0} t}
$$

where $\bar{n}$ is the mean number of photons of frequency $\nu_{0}$.

Since we ignore amplitude fluctuations, the phase $\theta$ performs a one-dimensional random walk around a

\footnotetext{
${ }^{2}$ For the expositions of the various schools of laser theory, see Haken (1970), Louisell (1973), and Sargent et al. (1974).
} 
circle, as indicated by Fig. 3. The quantum theory of the laser shows that the probability $P(\theta)$ of finding the phase $\theta$ obeys a phase diffusion equation of the FokkerPlanck type (Risken, 1984) with the diffusion constant $D=A /(4 \bar{n})$. Here $A$ denotes the linear gain. This diffusion causes a decaying average electric field

$$
\langle E(t)\rangle=\int d \theta P(\theta, t) E(\theta)=E_{0} e^{-D t},
$$

which gives rise to the laser linewidth.

This situation is analogous to ferromagnetism, where the magnetization of an open system (magnet) experiences the same kind of decay, albeit on a geological time scale. Measured in terms of the atomic and cavity lifetimes, the decay of $\langle E(t)\rangle$ is also very slow, typically estimated to take many minutes. Consequently, the laser/maser linewidth is much narrower than that of the atoms or molecules that drive it.

To many physicists steeped in the uncertainty principle, the maser's performance, at first blush, made no sense at all: Molecules spend so little time in the cavity of a maser, about one-ten-thousandth of a second, that it seemed impossible for the frequency of the radiation to also be narrowly confined. Townes recalls a conversation with Niels Bohr on a sidewalk in Denmark in which Bohr emphasized this very argument. After Townes persisted, he said: "Oh, well, yes, maybe you are right." But, the impression was that he was simply trying to be polite to a younger physicist.

\section{B. Squeezing and the correlated spontaneous emission laser}

Interferometers have great potential for highprecision measurements and tests of the foundations of physics. Examples include optical rotation sensors, such as fiber-optic or ring-laser gyroscopes (Chow et al., 1985), and probes of gravitomagnetic fields à la LenseThirring, and the search for gravitational waves (Schleich and Scully, 1984). In all of these examples the signal is encoded in the phase difference between two electromagnetic fields. Hence the fluctuations in the phases of the two fields determine the ultimate sensitivity of these devices.

In a passive interferometer, such as the fiber-optic laser gyro or a gravitational-wave detector, the laser is outside of the interferometer. The phase noise in the two arms of the interferometer, originating from spontaneous emission, is correlated and cancels since it comes from the same source. The only remaining noise is then due to the quantum nature of the radiation. It is the uncertainty $\Delta \theta$ of the laser field in phase as

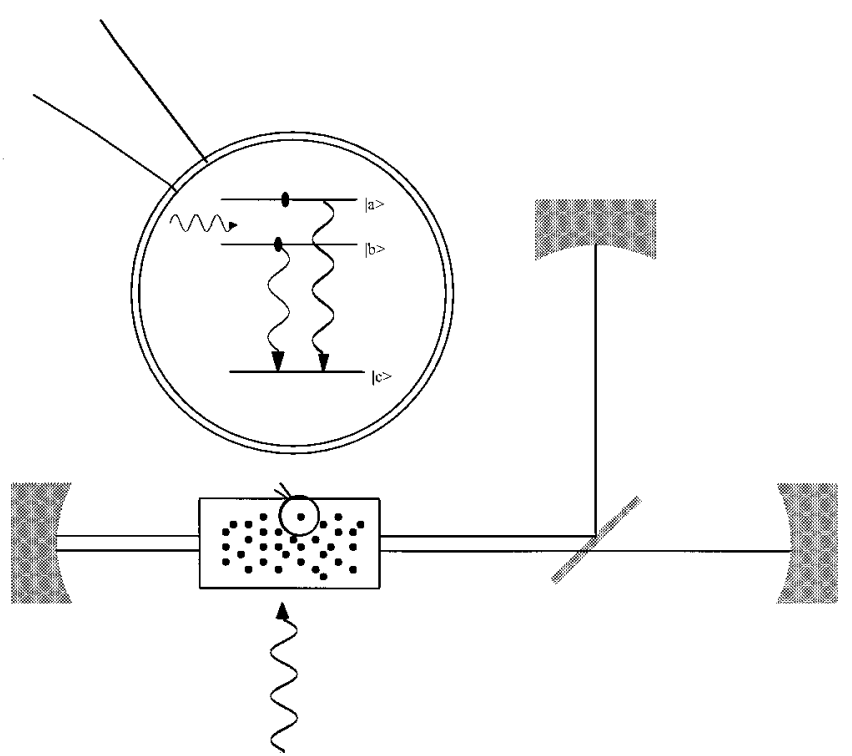

FIG. 4. Schematic setup of the correlated spontaneous emission laser (CEL) in the form of the quantum-beat laser. A microwave drives the two upper levels of a three-level atom. The two cavities, resonant with the two transitions to the ground state, are aligned in an orthogonal way as to be sensitive to gravitational waves or to rotation. Due to the coherence of the two upper states, the spontaneous emission in the two transitions is correlated. This leads to a dramatic suppression of the laser phase noise between the two fields and thus to a small Schawlow-Townes linewidth.

governed $^{3}$ by $\Delta n \Delta \theta \geqslant 1 / 2$. Since for a coherent state we have $\Delta n=\sqrt{\bar{n}}$ we find $\Delta \theta \cong 1 / 2 \sqrt{\bar{n}}$. However, when we use appropriately "squeezed light" we can achieve a situation such that $\Delta \theta \cong 1 / \bar{n}$ as shown by Caves (1981). In the mid-1980s the pioneering, squeezing experiments by the groups of R. Slusher and H. J. Kimble created a new and thrilling field. For a review of the activities in this arena, see Kimble and Walls (1987) and Grangier et al. (1995). A different method for obtaining $\Delta \theta$ $=1 /(2 \bar{n})$ has been discussed earlier by Serber and Townes (1960).

In an active device, such as the laser gyro, the laser medium is inside the ring cavity. Hence spontaneous emission of the atoms represents the ultimate limitation. Can we overcome this noise? The correlated spontaneous emission laser (CEL) (Scully, 1985), shown in Fig. 4, provides the definite answer, Yes!

The correlated spontaneous emission laser relies on a specially prepared lasing medium such that the noise in the relative phase angle of the emitted radiation is eliminated. For this purpose we use a three-level atom wherein the two transitions, from two excited states to a common ground state, drive a doubly resonant cavity.

\footnotetext{
${ }^{3}$ According to Serber and Townes (1960) the photon number and phase uncertainty relation reads $\Delta n \Delta \theta \geqslant 1 / 2$ rather than $\Delta n \Delta \theta=1$. This is illustrated by a specific example given by these authors, where $\Delta n \Delta \theta=1 / 2 \sqrt{(\bar{n}+1) / \bar{n}}$. For large $\bar{n}$ the right-hand side indeed reduces to $1 / 2$.
} 


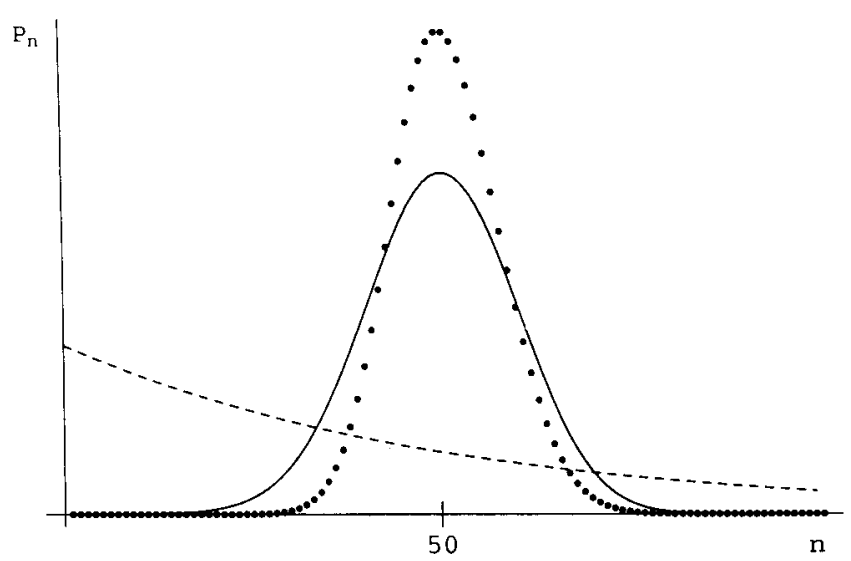

FIG. 5. Comparison between the photon statistics of a thermal state (dashed curve), coherent state (dotted curve) and a laser field (solid curve). In all three cases we have taken the average photon number $\bar{n}=50$. Whereas, the thermal distribution has its maximum at $n=0$ the Poissonian distribution of the coherent state and the laser photon statistics have their maximum at $n=\bar{n}$. We note however, that the photon statistics of the laser is broader than that of the coherent state.

They are strongly correlated by preparing the two upper levels in a coherent superposition. This is similar to quantum-beat experiments in which a strong external microwave signal produces coherent mixing and correlates the spontaneously emitted fields.

The correlated-spontaneous-emission-laser noise reduction was observed in a series of beautiful experiments (Winters et al., 1990, and Steiner and Toschek, 1995). However, the CEL concept is somewhat tricky, as expressed by a senior researcher in a discussion with Marlan O. Scully:

"The CEL is vastly more general than you think and vastly more trivial. It is simply due to the fact that the two lasers are locked together."

It is true that the CEL involves phase-locked lasers. However, there is more: The spontaneous emission events in the two transitions are correlated. This leads to dramatically different equations of motion for the phase difference: Whereas, in a phase-locked laser, the spontaneous emission noise is additive, in the CEL it enters in a multiplicative way (Schleich and Scully, 1988).

\section{Photon statistics}

As with the linewidth, so with the photon statistics; confusion abounded in the early days of the laser. Some people said that since "photons" are Bose-Einstein (BE) "particles" they must obey BE statistics. However, when we recall that the BE distribution applies to a system in thermal equilibrium and recognize that a laser is a system far away from thermodynamic equilibrium, we discover that this argument is false.

Indeed, the quantum theory of the laser predicts (Scully and Lamb, 1967) that the photon statistics of a laser, shown in Fig. 5, are substantially different from the photon statistics

$$
P_{n}=(1+\bar{n})^{-1}\left(\frac{\bar{n}}{1+\bar{n}}\right)^{n}
$$

of a thermal state or even from the Poissonian distribution (Glauber, 1964)

$$
P_{n}=\frac{\bar{n}^{n}}{n !} e^{-\bar{n}}
$$

of a coherent light beam. This feature has been verified by the groups of Tito Arecchi, Werner Martienssen, and Roy Pike in the early days of laser physics (Mandel and Wolf, 1970).

\section{One-atom masers/lasers and the mazer}

The one-atom maser ${ }^{4}$ or "micromaser" is an ideal testing ground for the quantum theory of the laser and extensions thereof. In the micromaser a stream of twolevel atoms passes through a superconducting cavity with a high-quality factor. The injection rate can be such that only one atom is present inside the resonator at any time. Due to the high-quality factor of the cavity, the radiation decay time is much larger than the characteristic time of the atom-field interaction. A field builds up inside the cavity when the mean time between the atoms injected into the cavity is shorter than the cavity decay time. Therefore a micromaser allows sustained oscillations with less than one atom on the average in the cavity.

The enormous progress in producing mirrors with almost 100 percent reflectivity has opened a new era in cavity quantum electrodynamics. Experiments, previously performed only in the microwave domain, have now been extended to the optical domain (Kimble et al., 1996). Moreover, an optical version of the one-atom maser, the so-called one atom laser (An et al., 1994), microlasers based on molecules (De Martini et al., 1993), and condensed matter systems (Yamamoto and Slusher, 1993), have been realized experimentally. For a more detailed discussion of laser technology we refer to the article by R. Slusher in this issue.

An interesting "spin-off" of the micromaser is its extension into the microwave amplification via $\mathrm{z}$-motioninduced emission of radiation (mazer) regime (Scully et al., 1996) when the kinetic energy is comparable to the interaction energy. Indeed, in this regime very slow (laser-cooled) atoms can be reflected from or tunnel through the cavity. In the process they undergo a new kind of induced emission, which is different from the stimulated emission of the ordinary micromaser regime with fast, that is, thermal atoms.

\footnotetext{
${ }^{4}$ For a review of the work of the Garching and Paris groups see Walther (1992) and Raithel et al. (1994), and Haroche and Raimond (1994) and Haroche (1998), respectively. For the theoretical work, see Meystre (1992) and Scully and Zubairy (1997).
} 

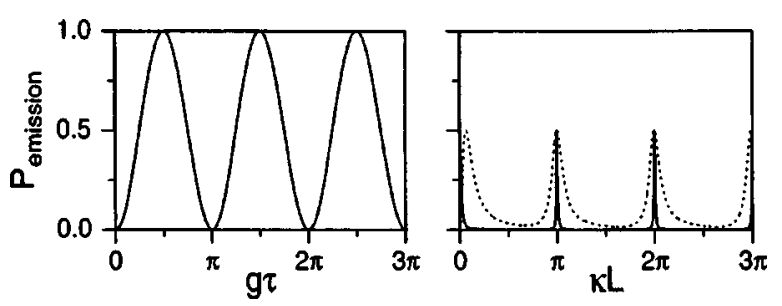

FIG. 6. Maser and Mazer compared and contrasted using the emission probability as a function of interaction time (left) or interaction length (right). A two-level atom of mass $M$ enters in its excited state a cavity which is initially in the vacuum state. On the left, the kinetic energy $(\hbar k)^{2} / 2 M$ is much larger than the interaction energy $\hbar g \equiv(\hbar \kappa)^{2} / 2 M$, that is, $k / \kappa=10$. On the right, it is smaller (dotted curve for $k / \kappa=0.1$ ) or much smaller (solid curve for $k / \kappa=0.01$ ) than $\hbar g$.

The difference between the classical and the quantum treatment of the center-of-mass motion comes out most clearly in the probability that an excited atom launched into an empty cavity will emit a photon. Figure 6 shows that when an integral number of half de Broglie wavelengths of the atomic center-mass-motion equals the cavity length, the atoms are transmitted through the cavity and new type of induced emission comes into play.

\section{E. The laser phase transition analogy}

We conclude this section by noting that in many ways the physics of the laser is analogous to that of a cooperative system near a phase transition. The root of the analogy is the fact that both the laser and the ferromagnet (or superconductor) are, to a conceptual idealization, mean-field or self-consistent field theories. In the many-body ferromagnet or superconductor problem, any given element of the ensemble sees all the other elements through the self-consistent mean field. For example, in the magnetic problem each spin communicates with all other spins through the average magnetization. Similarly, the atoms in the laser contribute to the total electric field by means of their induced dipole. This dipole is in turn induced by the mean electric field as contributed by all of the other atoms in the ensemble.

This analogy comes to light when we expand the density operator

$$
\rho=\int P(E)|E\rangle\langle E| d^{2} E
$$

of the laser field in terms of coherent states $|E\rangle$. The Glauber-Sudarshan $P$-distribution (Sargent et al., 1974) then reads (DiGiorgio and Scully, 1970; Graham and Haken, 1970)

$$
P(E)=\frac{1}{Z} e^{-G(E) / k_{L} \sigma},
$$

where

$$
G(E) \equiv \frac{A}{2}\left(\sigma-\sigma_{t}\right)|E|^{2}+\frac{B}{4} \sigma|E|^{4} .
$$

Here $A$ and $B$ are linear and nonlinear gain parameters, and $\sigma$ and $\sigma_{t}$ denote the inversion and the threshold inversion. The spontaneous emission rate $4 k_{L}$ per atom plays the role of the Boltzmann constant of equilibrium statistical mechanics.

This is to be compared to a ferromagnet. Here, in the Ginzburg-Landau approximation to the second-order phase transition, the free energy $F(M)$ reads

$$
F(M)=C\left(T-T_{c}\right) M^{2}+D T M^{4},
$$

where $C$ and $D$ are constants and $T_{c}$ is the Curie temperature.

\section{EXOTIC LASERS AND OUTLOOK}

In the following subsections, we briefly discuss three exotic laser systems - the free-electron laser, the laser without inversion, and the so-called atom laserillustrating in an impressive way that laser physics is still an evolving field. We do not concern ourselves with the details, but rather bring out the underlying physics of these devices. In the spirit of quantum controversy, we focus on the quantum aspects.

\section{A. The free-electron laser}

The free-electron laser (FEL) is an interesting device because it is largely free from the constraints imposed by the atomic medium of an ordinary laser. In a freeelectron laser, a relativistic electron beam interacts with a periodic static magnetic field called a wiggler (Brau, 1990). The electrons convert their kinetic energy into laser light. Indeed, the individual electrons passing through the magnetic field undergo transverse oscillations. Thus the electrons periodically accelerate and decelerate while they absorb and emit radiation. Depending on the wiggler length and the velocity of the electrons, we therefore have net gain or net loss.

We note, however, that the physical principle governing the FEL was initially strongly debated. It was first thought that FEL operation takes place only because of the quantum nature of the electron and photon. It was later shown that the FEL is a classical device (Hopf et al., 1976). The free-electron laser is therefore an excellent example of how the "photon-picture" can obscure the physics, which is electron bunching in a ponderomotive potential. The lasing process in a FEL may thus be compared with amplification and oscillation in electron tubes, which were characteristically used for radio frequencies before quantum electronics appeared on the scene.

\section{B. Lasers without inversion}

A laser seems to require population inversion in order to overcome absorption. Can we engineer a laser medium in which we cancel absorption but keep stimulated emission? Can we then lase without inversion? The answer is "Yes"! 
To understand this we again focus on a three-level atom. However, in contrast to the discussion of the quantum-beat laser of Sec. V.B, we now consider transitions between a single upper level and two lower levels. The absorption probability is then the coherent sum of the probability amplitudes of the two transitions. They can therefore cancel each other. This feature of vanishing absorption in this particular three-level configuration is yet another manifestation of quantum coherence. For a comprehensive discussion of these ideas we refer to Kocharovskaya and Khanin (1988), Harris (1989), and Scully et al. (1989). In contrast, in the emission process we end up in two different states; consequently, the two corresponding probabilities add up. This asymmetry between absorption and emission is the basis for the phenomenon of lasing without inversion.

We conclude by noting that many experiments have verified this and other related coherence effects, for example, electromagnetically induced transparency. However, this is not the place to go deeper into this exciting field. For an introduction and overview, we refer to Arimondo (1996) and Harris (1997).

\section{The atom laser}

Bose-Einstein condensation (BEC) of dilute $\mathrm{He}^{4}$ in a porous jell has been observed (Reppy, 1992). In addition, the pioneering experiments reporting BEC in lasercooled atoms have ushered in a new era in the study of $\mathrm{BEC}$ in dilute low-temperature gases. In particular, the striking experimental demonstration of interference between two condensates and the time evolution of a condensate provide incontrovertible evidence for BEC. For a summary of these experiments we refer to the article by Wieman et al. in the present issue.

They have clearly demonstrated that Bosonicstimulated emission is important in the process. This has suggested a comparison with the laser and coined the name "atom laser" for certain kinds of coherent atom beams. The MIT group has already realized experimentally such an atom laser. In this context it is interesting to note that an analog of the semiclassical theory of the optical laser can describe the essential features of this device (Kneer et al., 1998). This is only one of many applications of laser concepts to BEC, which will continue to be a low-temperature "hot topic."

\section{ACKNOWLEDGMENTS}

We thank M. Hug, S. Meneghini, P. Meystre, and H. Walther for their comments on and help with this manuscript. W.E.L. expresses his appreciation to the Humboldt Stiftung. The work of W.P.S. is partially supported by the Deutsche Forschungsgemeinschaft, and that of M.O.S. by the Office of Naval Research, the National Science Foundation, and the Welch Foundation.

\section{REFERENCES}

Arimondo, E., 1996, in Progress in Optics, edited by E. Wolf (North-Holland, Amsterdam), Vol. 35, p. 257.

An, K., J.J. Childs, R.R. Desari, and M.S. Feld, 1994, Phys. Rev. Lett. 73, 3375.

Basov, N.G., and A.M. Prokhorov, 1955, Dokl. Akad. Nauk 101, 47.

Basov, N.G., B.M. Vul, and Yu.M. Popov, 1959, Zh. Eksp. Teor. Fiz., 37, 587 [Sov. Phys. JETP 10, 416].

Becker, G.E., and S.H. Autler, 1946, Phys. Rev. 70, 300.

Bloembergen, N., 1956, Phys. Rev. 104, 324.

Brau, C.A., 1990, Free-Electron Lasers (Academic Press, Orlando).

Caves, C.M., 1981, Phys. Rev. D 23, 1693.

Chow, W.W., J. Gea-Banacloche, L. Pedrotti, V. Sanders, W. Schleich, and M.O. Scully, 1985, Rev. Mod. Phys. 57, 61.

Cleeton, C.E., and N.H. Williams, 1934, Phys. Rev. 45, 234.

Combrisson, J., A. Honig, and C.H. Townes, 1956, Phys. Rev. 242, 2451.

De Martini, F., M. Marrocco, P. Mataloni, and D. Murra, 1993, J. Opt. Soc. Am. B 10(2), 360.

Dennison, D.M., and G. E. Uhlenbeck, 1932, Phys. Rev. 41, 313.

DiGiorgio, V., and M.O. Scully, 1970, Phys. Rev. A 2, 1170.

Glauber, R., 1964, in Quantum Optics and Electronics, edited by C. DeWitt, A. Blandin, and C. Cohen-Tannoudji (Gordon and Breach, New York).

Gordon, J.P., H.J. Zeiger, and C.H. Townes, 1954, Phys. Rev. 95, 282L.

Gordon, J.P., H.J. Zeiger, and C.H. Townes, 1955, Phys. Rev. 99, 1264.

Graham, R., and H. Haken, 1970, Z. Phys. 237, 31.

Grangier, P., E. Giacobino, and P. Tombesi, 1995, Eds. Nonclassical Light, J. Quantum Semiclass. Opt. 7, 499.

Haken, H., 1970, Laser Theory in Encyclopedia of Physics Vol. XXV/2c, edited by S. Flügge (Springer, Berlin).

Hall, R.N., G.E. Fenner, J.D. Kingsley, T.J. Soltys, and R.O. Carlson, 1962, Phys. Rev. Lett. 9, 366.

Harnwell, G. P., 1938, Principles of Electricity and Electromagnetism (McGraw-Hill, New York).

Haroche, S., 1998, Phys. Today 51(7), 36.

Haroche, S., and J.-M. Raimond, 1994, in Cavity Quantum Electrodynamics, Adv. At. Mol., and Opt. Phys., Suppl. 2, edited by P.R. Berman (Academic Press, New York), p. 123. Harris, S.E., 1989, Phys. Rev. Lett. 62, 1033.

Harris, S.E., 1997, Phys. Today 50(7), 36.

Hopf, F.A., P. Meystre, M.O. Scully, and W.H. Louisell, 1976, Phys. Rev. Lett. 37, 1342.

Javan, A., W.R. Bennett, and D.R. Herriott, 1961, Phys. Rev. Lett. 6, 106.

Kimble, H.J., Q.A. Turchette, N.Ph. Georgiades, C.J. Hood, W. Lange, H. Mabuchi, E.S. Polzik, and D.W. Vernooy, 1996, in Coherence and Quantum Optics VII, edited by J.H. Eberly, L. Mandel, and E. Wolf (Plenum Press, New York), p. 203.

Kimble, H.J., and D.F. Walls, 1987, Eds., Squeezed States of the Electromagnetic Field, J. Opt. Soc. Am. B 4, 1450.

Kneer, B., T. Wong, K. Vogel, W.P. Schleich, and D.F. Walls, 1998, Phys. Rev. A 58, 4841.

Kocharovskaya, O., and Ya. I. Khanin, 1988, Pis'ma Zh. Éksp. Teor. Fiz. 48, 581 [JETP Lett. 48, 630].

Ladenburg, R., 1933, Rev. Mod. Phys. 5, 243.

Lamb, W.E., 1946, Phys. Rev. 70, 308. 
Lamb, W.E., 1960, in Lectures in Theoretical Physics, edited by W.E. Brittin and B.W. Downs (Interscience Publishers, New York), Vol. 2.

Lamb, W.E., 1964a, in Quantum Optics and Electronics, edited by C. DeWitt, A. Blandin, and C. Cohen-Tannoudji (Gordon and Breach, New York).

Lamb, W.E., 1964b, Phys. Rev. 134, A1429.

Lamb, W.E., 1984, IEEE J. Quantum Electron. 20, 551.

Lamb, W.E., 1995, Appl. Phys. B: Lasers Opt. 60, 77.

Lamb, W.E., and R.C. Retherford, 1950, Phys. Rev. 79, 549.

Lax, M., 1965, in Proceedings of the International Conference on Quantum Electronics, Puerto Rico 1965, edited by P.L. Kelley, B. Lax, and P. Tannenwald (McGraw Hill, New York).

Lewis, G.N., 1926, Nature (London) 118, 874.

Louisell, W.H., 1973, Quantum Statistical Properties of Radiation (Wiley, New York).

Maiman, T.H., 1960, Nature (London) 187, 493.

Mandel, L., and E. Wolf, 1970, Selected Papers on Coherence and Fluctuations of Light (Dover, New York).

McFarlane, R.A., W.R. Bennett, and W.E. Lamb, 1963, Appl. Phys. Lett. 2, 189.

Meystre, P., 1992, in Progress in Optics, edited by E. Wolf (North-Holland, Amsterdam), Vol. 30, p. 263.

Raithel, G., C. Wagner, H. Walther, L. Narducci, and M.O. Scully, 1994, in Cavity Quantum Electrodynamics, Adv. At. Mol., and Opt. Phys., Suppl. 2, edited by P.R. Berman (Academic Press, New York), p. 57.

Reppy, J.D., 1992, J. Low Temp. Phys. 87, 205.

Risken, H., 1965, Z. Phys. 186, 85.

Risken, H., 1984, The Fokker Planck Equation (Springer, Heidelberg).

Sargent, M., M.O. Scully, and W.E. Lamb, 1974, Laser Physics (Addison-Wesley, Reading).

Schawlow, A.L., and C.H. Townes, 1958, Phys. Rev. 112, 1940. Schleich, W., and S.M. Barnett, 1993, Quantum Phase and Phase Dependent Measurements, Phys. Scr. T48, 3.
Schleich, W., and M.O. Scully, 1984, in Modern Trends in Atomic Physics, edited by G. Grynberg, and R. Stora (NorthHolland, Amsterdam).

Schleich, W., and M.O. Scully, 1988, Phys. Rev. A 37, 1261.

Scully, M.O., W.E. Lamb, and M.J. Stephen, 1965, in Proceedings of the International Conference on Quantum Electronics, Puerto Rico, 1965, edited by P.L. Kelley, B. Lax, and P. Tannenwald (McGraw Hill, New York).

Scully, M.O., and W.E. Lamb, 1967, Phys. Rev. 159, 208.

Scully, M.O., 1985, Phys. Rev. Lett. 55, 2802.

Scully, M.O., G.M. Meyer, and H. Walther, 1996, Phys. Rev. Lett. 76, 4144.

Scully, M.O., S.-Y. Zhu, and A. Gavrielides, 1989, Phys. Rev. Lett. 62, 2813.

Scully, M.O., and M.S. Zubairy, 1997, Quantum Optics (Cambridge University Press, Cambridge).

Serber, R., and C. H. Townes, 1960, Quantum Electronics, edited by C. H. Townes (Columbia University, New York).

Shimoda, K., T.C. Wang, and C.H. Townes, 1956, Phys. Rev. 102, 1308.

Shimoda, K., H. Takahasi, and C.H. Townes, 1957, J. Phys. Soc. Jpn. 12, 686.

Siegman, A.E., 1986, Lasers (University Science Books, Mill Valley).

Steiner, I., and P.E. Toschek, 1995, Phys. Rev. Lett. 74, 4639.

Szöke, A., and A. Javan, 1963, Phys. Rev. Lett. 10, 521.

Tolman, R.C., 1924, Phys. Rev. 23, 693.

Townes, C.H., 1946, Phys. Rev. 70, 665.

Townes, C.H., 1999, How the Laser Happened; Adventures of a Scientist (Oxford University, Oxford).

Van Vleck, J.H., and V.F. Weisskopf, 1945, Rev. Mod. Phys. 17, 227.

Walther, H., 1992, Phys. Rep. 219, 263.

Weber, J., 1953, IRE Trans. Electron Devices 3, 1.

Winters, M.P., J.L. Hall, and P.E. Toschek, 1990, Phys. Rev. Lett. 65, 3116.

Yamamoto, Y., and R.E. Slusher, 1993, Phys. Today 46(6), 66. 